Aims

- To develop a six week OT-led therapeutic creative group, based on creating a legacy frame

- Enabling patients to use creative activities to explore significant life events

- To evaluate the group using an outcome measure and patient feedback.

Methods A legacy project was developed that explored therapeutic aims. Patients were invited to select a theme for their project and bring meaningful items to the group to use in their box frame. The FACIT Spiritual Well-Being (FACIT-Sp) and a non-standardised outcome measure were selected to evaluate the group.

Results Five patients completed the group, one dropped out as they felt unable to cope with the emotions they experienced and one patient died while completing their project. Patients chose legacy projects around topics of travel, career achievements, family, marriage and hobbies. Themes explored through facilitated group discussion as part of the project included: grief, living with a life-threatening condition, death of parents, divorce, anger, reconciliation, children, self-esteem, fear of trusting others and guilt.

Patient feedback from the sessions included quotes such as

'I feel safe to be me, to discuss and express how I am really feeling'

'very inspired'

'I would like my family to know more about me'

FACIT-Sp demonstrated little or no change.

Conclusion Qualitative feedback was most helpful in demonstrating the impact of the group. A different outcome measure may have been more sensitive to change. The group provided a safe space to explore difficult topics.

\section{P-185 EVALUATING THE IMPACT OF A CAFÉ STYLE SOCIAL DROP IN GROUP}

Katy Talbot-Vaux, Emily Stowe, Sarah Thompson. St Clare Hospice, Hastingwood, UK

\subsection{6/bmjspcare-2018-hospiceabs.210}

Background It is estimated there are 1.2 million lonely older adults in the UK (Campaign to End Loneliness). Increasing numbers of patients who were completing their day therapy programme reported they felt isolated and wished to continue linking into the hospice. As part of restructuring of Day Therapy it was recognised that social isolation was impacting patients at the hospice. A café style drop-in group was introduced which runs weekly.

Aims

- To introduce a café style social drop in group

- To evaluate the group using an outcome measure and patient feedback

- To reduce social isolation in patients attending the group

- Use the café as way to introduce people to the hospice.

Methods We introduced a therapy assistant and volunteer-led café for patients and their friends/families. Volunteer transport was available. The De Jong Gierveld Loneliness Scale (long version) (DJGLS) outcome measure was selected to evaluate the group and is completed as a weekly questionnaire by attendees. Qualitative feedback was also gained from the attendees.
Results An average of 16 people have attended the group on a weekly basis. The majority of people attending come each week, however for some people attending it has been their first contact with the hospice. More than $80 \%$ of the attendees are men. As the service is new, we do not yet have sufficient data from the DJGLS to statistically analyse, however, this will be completed by November 2018. Feedback from patients is positive and includes:

'I always love coming here, I go home refreshed with the love'

'Fantastic to look forward to each week'

'Supportive'

The cost per session, has been calculated at $£ 71$, approximately $£ 4.50$ per person attending.

Conclusion The group has provided a low cost, 'light touch' social support service. Qualitative feedback has suggested that the group is providing appropriate support.

\section{P-186 SETTING OFF ON OUR JOURNEY TO WIDER AND EARLIER HOSPICE ENGAGEMENT}

Paula Bavetta. Isabel Hospice, Welwyn Garden City, Herts

\subsection{6/bmjspcare-2018-hospiceabs.211}

Background Our statistics showed that the average length of time from referral to death in March 2017 was 1-2 months. This represented too many patients missing out on support and planned end of life care. We hypothesised that by engaging with more people, earlier we might reduce the stigma around engagement with hospice services.

Aims Our aim was to increase the number of people accessing the Living Well Services over a six-month period.

Methods After considering which activities attracted more referrals and listening to our users we relaunched our original day services as 'Living Well Services'. The programme of activities offered was enhanced to offer more of our popular activities such as physiotherapy supervised exercise groups, Fatigue and breathlessness courses, Creative Cafes, singing and Tai Chi. We used community collaborations to deliver some of these activities. We also complemented our day service with a support group for patients with complex neurodegenerative conditions. We presented our new service to community colleagues, targeting disease specific groups such as pulmonary rehab courses where we delivered education on 'Living with Long Term Conditions', the Heart Failure CNS team at the acute trusts, as well as preestablished links with the local Navigator for Complex Neuro. We also opened all our Living Well services to selfreferrals and improved our website to encourage engagement.

Results Over the period October 2017 to March 2018 the total number of attendances at the Living Well services increased from 340 to 667 representing a 96\% increase. This included seven self-referrals. The caseload increased from 131 to 186 , a $42 \%$ increase. Our non -cancer referrals increased from 38\% in October 2017 to 48\% in March 2018.

Conclusions We will review first referral to death times in a year to confirm that the significant increase in earlier engagement carries through. 